\title{
Understanding complex matter from simple packing models
}

\author{
T. Aste, G. Delaney and T. Di Matteo \\ Department of Applied Mathematics, The Australian National University, 0200 Canberra, \\ ACT, Australia.
}

\begin{abstract}
By pouring equal balls into a container one obtains disordered packings with fascinating properties which might shed light on several elusive properties of complex materials such as amorphous metals or colloids. In any real experiment with equal-sized spheres one cannot reach packing fractions (fraction of volume occupied by the spheres respect to the total volume, $\rho$ ) below the Random Loose Packing limit (RLP, $\rho \sim 0.555$ ) or above the Random Close Packing limit (RCP, $\rho \sim 0.645$ ) unless order is externally induced. What is happening at these two limits is an open unanswered question. In this paper we address this question by combining statistical geometry and statistical mechanics methods. Evidences of phase transitions occurring at the RLP and RCP limits are reported.
\end{abstract}

PACS: 45.70.-n Granular Systems 45.70.Cc Static sandpiles; Granular Compaction 81.05.RmPorous materials; granular materials

\section{INTRODUCTION}

Equal sized spheres are the simplest three-dimensional geometrical objects. However, despite such simplicity very little is known about the structures that they form when they pack together. In 1611 Kepler conjectured that equal spheres cannot be packed denser than the packing fraction $\rho=\pi / \sqrt{18} \sim 0.74$ which is achieved by stacking hexagonally closed packed layers as, for instance, in fcc and hcp crystalline packings. ${ }^{1}$ For Kepler himself it was just 'obious' that denser configurations cannot exist, and he left the proof to posterity. Surprisingly, it took more than three hundred years to prove such an intuitive conjecture and this is the only known exact result in this matter. And, even the correctness of this proof, is still under scrutiny. Understanding packing properties is of central importance in a large number of fields from cosmology to condensed matter physics. In particular, disordered packings are central in the study of so-called 'granular materials'. The term granular matter refers to particle systems in which the sizes of the constituents are large enough such that they are not subject to thermal motion fluctuations. Thus, the typical lower grain size is around $1 \mu m$, but the largest grains can have sizes on tectonic scales. These materials are everywhere and the capability to handling, processing, storing and producing them is of paramount importance. Granular materials are central in a very wide range of domains from agriculture to pharmaceuticals. But, despite such a central role in most fields of human activity and their ubiquitous presence in scientific research areas, a complete understanding of their behaviour and properties remain elusive. Granular materials differ from all the other materials: they can flow like liquids under some conditions, yet act as solids under others. Their intrinsic complexity is a consequence of the fact that they are composed of many pieces that can assemble in large structures that move collectively. Therefore the packing structure plays a central role in determining their properties. The understanding of these disordered systems requires new paradigms and tools beyond the traditional domains of solid state physics, engineering and material sciences. The study of granular materials forces scientists to rethink established classifications of matter and to reformulate statistical mechanics in a new context. These studies are producing new tools that are advancing the understanding of large class of complex materials from composites to foams.

Since the earliest studies of granular materials it was evident that one of the key quantities which affects the system's properties is the packing fraction. It is well known since ancient times that different actions and variations on a given action can generate packings with different packing fractions. Typically, in experiments, such 'actions' consist of tapping the system with vertical vibrations, shearing, rotating the container or pouring the grains into the container. In times when grain was sold by volume, the preparation protocol to achieve a

Complex Systems II, edited by Derek Abbott, Tomaso Aste, Murray Batchelor, Robert Dewar,

Tiziana Di Matteo, Tony Guttmann, Proc. of SPIE Vol. 6802, 68020E, (2008)

0277-786X/08/\$18 - doi: $10.1117 / 12.759030$

Proc. of SPIE Vol. 6802 68020E-1 
dense packing was very important. ${ }^{2}$ This is even reported in the gospel as an example of good measure: "Give, and it shall be given to you. Good measure, pressed down, shaken together, running over, will be put into your lap." (Luke 6:38). Indeed, depending on the system handling, one can have variations in packing fraction up to $15 \%$ within the two limits $\rho \sim 0.555$ and $\rho \sim 0.645$ which are commonly refereed to Random Loose Packig (RLP) and Random Close Packing (RCP) limits. For instance, by using the fluidized bed technique ${ }^{3,4}$ one can obtain packing fractions in the whole spectrum from 0.55 to 0.645 by varying the intensity of the flow pulses. In numerical simulations many other possibilities are available.

One of the scientists who first investigated the microscopic nature of granular packing was J.D Bernal, who in a stream of papers concerning the "structure of liquids" reported some of the most important features of the structural organization of disordered sphere packings. ${ }^{2,5,6}$ It was Bernal who pointed out that disordered packing of equal spheres cannot overcome the RCP limit. Fascinated by the simultaneous simplicity and complexity of these systems, he asked the following question: "Science is measurement, but what is a good measure?". Indeed, he was aware that depending on the kind of external actions the system will result in different packing fractions. However, he also observed that for a given external action the system produces configurations with very similar and reproducible packing fractions which vary in a very narrow range within $0.5 \%$.

In this paper we show that the study of the fluctuations of such reproducible packing fractions can shed light on the origin of the RLP and RCP limits. Indeed, from a statistical mechanics perspective such fluctuations are a measure of the way in which the system is exploring the accessible phase-space under a given external action. Granular materials are particle systems in which the sizes of the constituents are large enough such that they are not subject to thermal motion fluctuations. Therefore, a direct application of a thermodynamical theory is not straightforward. However, in recent years several extensions of classical statistical mechanics approaches have been proposed for these systems. ${ }^{7-15}$ In this paper we use a statistical mechanics approach to relate the fluctuations of the packing fraction with changes in the system's structural organisation and find signatures of phase transitions. In his "Bakerian lecture", Bernal explained that in such systems there are two fundamental questions to be addressed: 1) "What is the structure?" and 2) Why has it got this structure?". And he resolved that the answer must be searched by two means: 1) Statistical Geometry and 2) Statistical Mechanics. By following his footsteps, in this paper we use a geometrical analysis and a statistical mechanics approach to understand what is happening at the RLP and RCP limits.

\section{MATERIALS AND METHODS}

\subsection{Experiments}

The experimental results reported in this paper concern experiments from the AAS database of disorder packings. ${ }^{16}$ Specifically, we investigate the six samples A-F described in details in. ${ }^{17-19}$ They are dry packings of acrylic mono-sized spherical beads prepared with different methods in a range of packing fractions between 0.58 and 0.64 .

\subsection{Numerical simulations}

We also generate a set of packings by means of an event-driven molecular dynamic simulation of hard spheres which uses a modified Lubachevsky-Stillinger algorithm. ${ }^{20-22}$ The algorithm starts from random points in space which grow uniformly into non-overallping spheres with the sphere positions evolving in time according to Newtonian dynamics. The algorithm uses a cubic box and periodic boundary conditions. The simulation is ended when the sphere sizes cannot be increased any longer and a 'jammed' state with diverging collision rate is reached. Large expansion rates produce jammed configurations with low packing fractions whereas slower growth rates leads to larger packing fractions. The least dense jammed configurations have packing fractions $\rho \sim 0.555$ which correspond to the RLP limit. On the other hand, for very slow rates, crystalline nuclei with large packing fractions (up to the limit $\sim 0.74$ ) are formed. In our simulations, by varying the growth relate between 500 and 0.00001, we generate jammed configurations with packing fractions between 0.56 and 0.65. We also generate non-jammed configurations in the range of packing fractions between 0.1 and 0.55 by keeping 
the growth rate at 0.001 and arresting the simulation once the desired packing fraction is reached. In our investigation each sample contains 10000 spheres.

\section{GEOMETRICAL STUDY OF A STRUCTURAL TRANSITION}

It has been pointed out in ${ }^{23}$ that the 'common-neighbor analysis of structure', first introduced by Clarke and Jónsson in,${ }^{24}$ is a very powerful method to detect structural organization. In such a construction one associates a 'bond' to couples of neighbouring spheres with centers within a given threshold radial distance, then retrieving the set of all all the common bonded neighbors of two bonded spheres. In this paper we choose a threshold distance of 1.255 sphere diameter. Such a distance coincides with the one used by Hales to individuate 'near' spheres in his recent proof of the Kepler's conjecture ${ }^{1}$ and it was also used recently by ${ }^{25}$ in the geometrical study of the RCP limit. This is a rather convenient distance: not too small in order to have little sensitivity to local rearrangements, and not too large, in order to avoid an averaging out of local properties.

We calculate the fractions $p(q)$ of couples with $q$ common neighbors respect to the total number of couples. Figure 1 shows the results of such analysis performed on 15 numerical simulations and on the 6 experiments A-F. Only fractions with $q=2,3,4,5$ (which have the largest statistical weight) are shown. The figure reveals a very good agreement between simulations and experiments indicating that the structural properties have little sensitivity to the preparation method and to the physical characteristics of the grains. Conversely the figure reveals a clear universal dependence of the structural properties on the packing fraction $\rho$ with opposite trends for $p(2), p(3)$ and $p(4), p(5)$.

A very clear signature that something is occurring in the structure around the Random Loose Packing limit is revealed by the sharp changes in the behaviors of $p(2)$ and $p(4)$ occurring between $\rho=0.55$ and $\rho=0.56$. Some evidence of the onset of a different regime can also be observed from the behavior of $p(3)$ and $p(5)$ around the Random Close Packing limit $(\rho \sim 0.645)$. Such a change must be due to the fact that in the crystalline phase (which, in the simulated samples, begins to nucleate above the RCP limit) there are no configurations with 3 or 5 common neighbors. Indeed, any closed packed phase, made by stacking hexagonal layers of spheres (Barlow packings ${ }^{1}$ ), can admit only 2 or 4 common neighbors.

In order to better visualize any structural transition, in Fig.2 we plot the ratio:

$$
\Delta=\frac{p(3) p(5)}{p(2) p(4)}
$$

which is a 'signature' of disordered arrangements weighting configurations which are impossible in crystalline packings (3 and 5 common neighbors) against configurations that instead are common in crystals (2 and 4 common neighbors). The plot of $\Delta$ reveals two sharp transitions occurring respectively at the RLP and at the RCP limits.

We have therefore acquired our first evidence that there are sharp structural changes occurring at the RLP and RCP limits. Now let us establish if these geometrical changes are associated with other changes in the statistical mechanical properties of these systems.

\section{A STATISTICAL MECHANICS STUDY OF A PHASE TRANSITION}

As discussed in the introduction, different preparation procedures and different experiments can result in granular packings with different total occupied volumes $V$ (or equivalently different packing fractions $\rho=$ $\pi N d^{3} /(6 V)$, where $N$ is the number of spheres and $d$ their diameters). Here we are interested in the properties of the set of all the possible total volumes (packing fractions) which can be reached as results of a chosen system's preparation technique.

Any classical statistical mechanics theory will typically yield to an expression for the probability distribution of the volume fluctuations at equilibrium of the following form: ${ }^{4,26}$

$$
p_{\infty}(V)=\frac{\Omega(V) e^{-V / \chi}}{\sum_{V^{\prime}} \Omega\left(V^{\prime}\right) e^{-V^{\prime} / \chi}} ;
$$

Proc. of SPIE Vol. 6802 68020E-3 


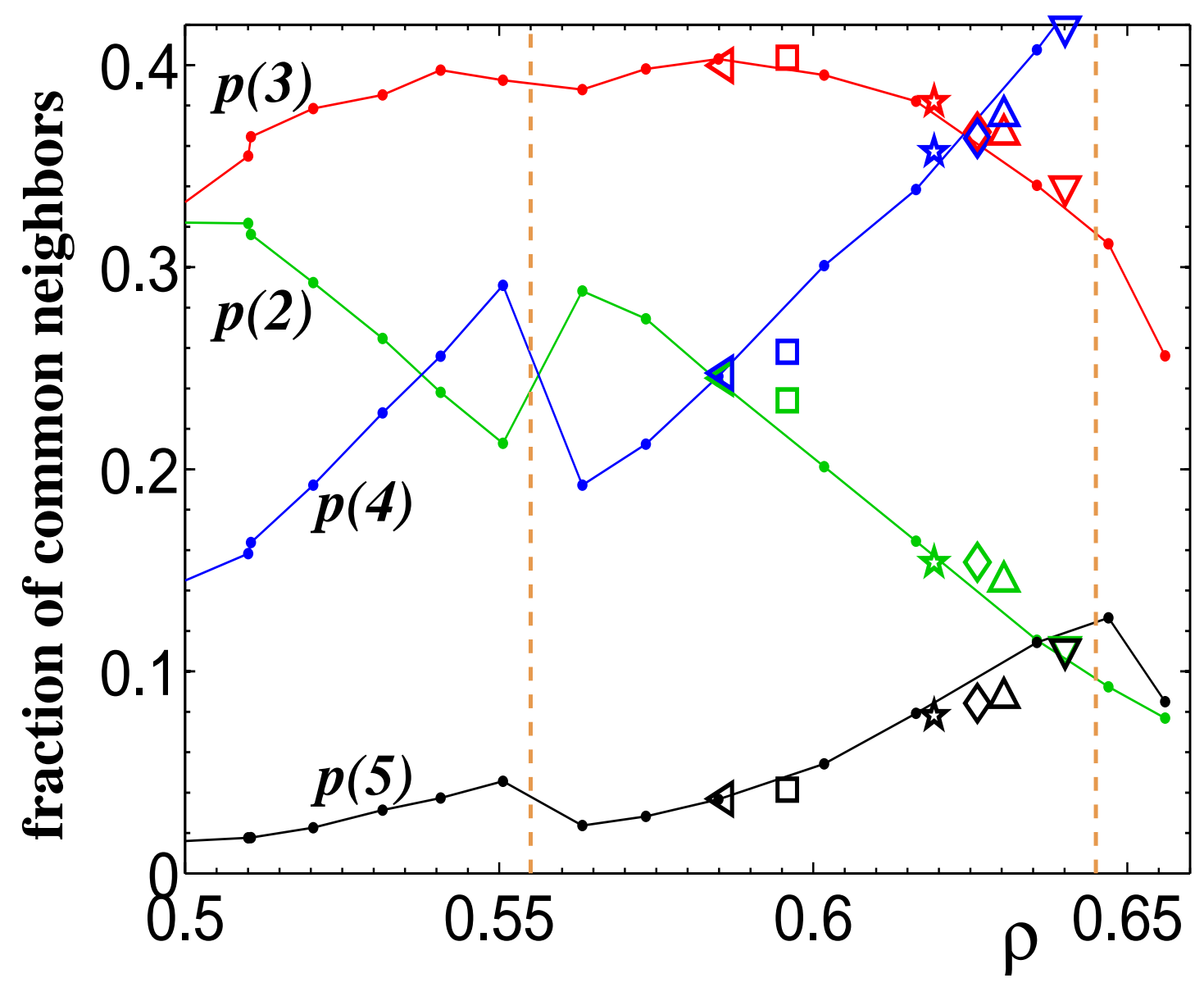

Figure 1. Fraction of common neighbors calculated from numerical simulations (dots and lines) and from experiments with dry acrylic spherical beads A $(\triangleleft)$, B $(\square)$, C $(\star)$, D $(\diamond)$, E $(\triangle)$ and F $(\nabla)$ vs. packing fraction $(\rho)$. Spheres are considered as neighbors if they stay within a threshold distance of 1.255 sphere diameters. The two vertical lines are respectively at $\rho=0.555$ and $\rho=0.645$ marking the RLP and RCP packing fractions. 


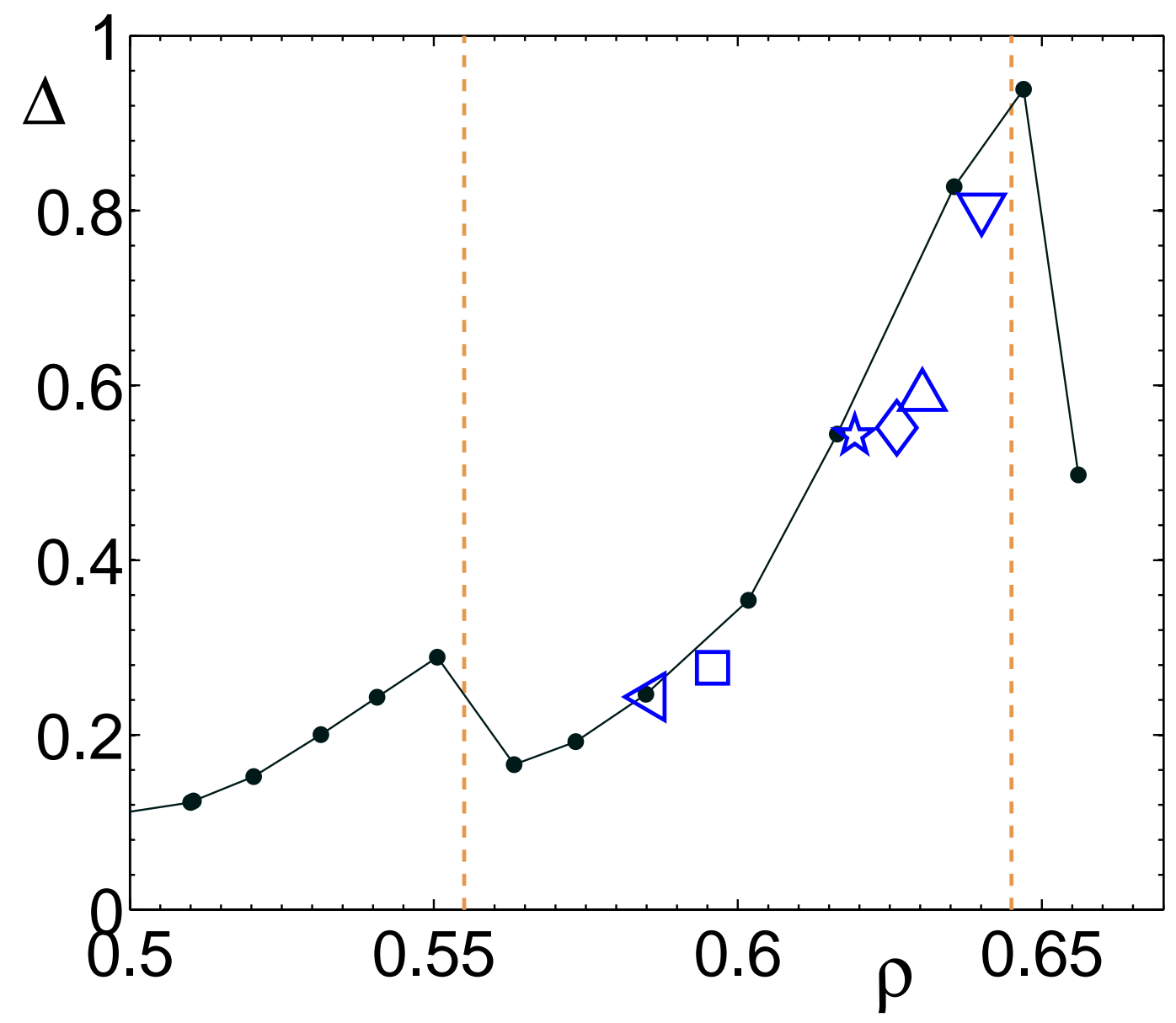

Figure 2. The ratio $\Delta=(p(3) p(5)) /(p(2) p(4))$ vs. $\rho$ reveals very sharp changes at the packing fractions corresponding to the Random Loose Packing and Random Close Packing limits (the two vertical lines). The dots (connected by straight lines) refer to numerical simulations and the symbols to experiments with dry acrylic spherical beads A $(\triangleleft)$, B $(\square)$, C $(\star), \mathrm{D}(\diamond), \mathrm{E}(\triangle)$ and $\mathrm{F}(\nabla)$. 
where $\Omega(V)$ is the number of microscopic states which are classifiable under the same (coarse grained) state with volume $V$. The quantity $\chi^{-1}$ is an intensive variable which is determined by the constraint on the average volume:

$$
\bar{V}=\langle V\rangle=\sum_{V} V p_{\infty}(V)
$$

A derivation of Eq.2 from a minimal set of statistical arguments is provided in; ${ }^{4}$ whereas a complete deductive statistical mechanics derivation is given in. ${ }^{26}$

The challenge is to compute the number of equilibrium configurations $\Omega(V)$ associated with states which occupy a total volume $V$ under a given system preparation. To this end we can image that the whole system is made of a number $k$ of 'elementary cells' $\left\{\mathbf{c}_{1}, \ldots, \mathbf{c}_{k}\right\} .{ }^{4,26}$ Given such cellular partition, $\Omega(V)$ can be computed exactly, under the two following assumptions: (1) these cells can have arbitrary volumes above a minimum value $v_{\text {min }}$, under the sole condition that the whole system must occupy a total volume $V ;(2)$ all the cell-properties $\mathbf{c}_{i}$ are either completely determined by their volumes $v_{i}$ or they are independent from $v_{i}$. In this case, we have

$$
\begin{aligned}
& \Omega(V)=\left(\frac{1}{\Lambda^{3}}\right)^{k} \int_{v_{\text {min }}}^{V} d v_{1} \int_{v_{\text {min }}}^{V} d v_{2} \ldots \int_{v_{\text {min }}}^{V} d v_{k} \\
& \delta\left(v_{1}+v_{2}+\ldots+v_{k}-V\right)=\frac{\left(V-k v_{\text {min }}\right)^{k-1}}{\Lambda^{3 k}(k-1) !},
\end{aligned}
$$

with $\Lambda$ a constant analogous to the Debye length. Substituting into Eq.2, and by using Eq.3 we obtain

$$
\chi=\frac{\left(\bar{V}-k v_{\min }\right)}{k}
$$

and

$$
p_{\infty}(V)=f(V, k)=\frac{k^{k}}{\Gamma(k)} \frac{\left(V-V_{\min }\right)^{(k-1)}}{\left(\bar{V}-V_{\min }\right)^{k}} \exp \left(-k \frac{V-V_{\min }}{\bar{V}-V_{\min }}\right)
$$

with $V_{\min }=k v_{\min }$. The function $f(V, k)$ is the probability density function to find a packing of $k$ cells occupying a volume $V$, when the system is subject to an external action that produces an average occupied volume $\bar{V}$. Note that Eq.4 is valid for any $k$ and it holds even in the limit $k=1$. Indeed, the observable system can be any arbitrary sub-set of a larger system. Moreover, the experiment can be performed either on several different independent systems or equivalently on several non-iteracting sub-sets of a large system. Eq.6 is a Gamma distribution in the variable $V-V_{\min }$; it is characterized by a 'shape' parameter $k$ and a 'scale' parameter $\chi{ }^{27}$ For this distribution the average volume $\langle V\rangle$ coincides with $\bar{V}$ and the variance is

$$
\sigma_{v}^{2}=\frac{\left(\bar{V}-V_{\min }\right)^{2}}{k}
$$

This last relation is very useful because it provides a practical means to evaluate $k$ from a set of volume measurements: $k=\left(\bar{V}-V_{\min }\right)^{2} / \sigma_{v}^{2}$.

\section{GRANULAR TEMPERATURE, FLUCTUATION RELATION AND SPECIFIC HEAT}

Equation 6 predicts that the statistical distribution of the volume fluctuations depends only on the parameter $k$ which counts the number of elementary cells in the system. Let us better understand the physical and statistical mechanics meaning of such a quantity. Following Edward's ideas, ${ }^{7,8}$ in granular systems a 'granular temperature' can be inferred from an analogy with the thermodynamical relation $\beta=1 /\left(k_{B} T\right)=$ $\partial($ Entropy $) / \partial($ Energy $),{ }^{7,8}$ by susbstituting the volume to the role played by the energy in thermodynamical systems. In the present approach we can write the 'statistical entropy' (or Gibbs entropy) for an ergodic set $Z$ characterized by and average volume $\bar{V}$, as: ${ }^{26}$

$$
S(Z)=-\sum_{V \in Z} p_{\infty}(V) \log p_{\infty}(V)+\sum_{V \in Z} p_{\infty}(V) S(V)
$$




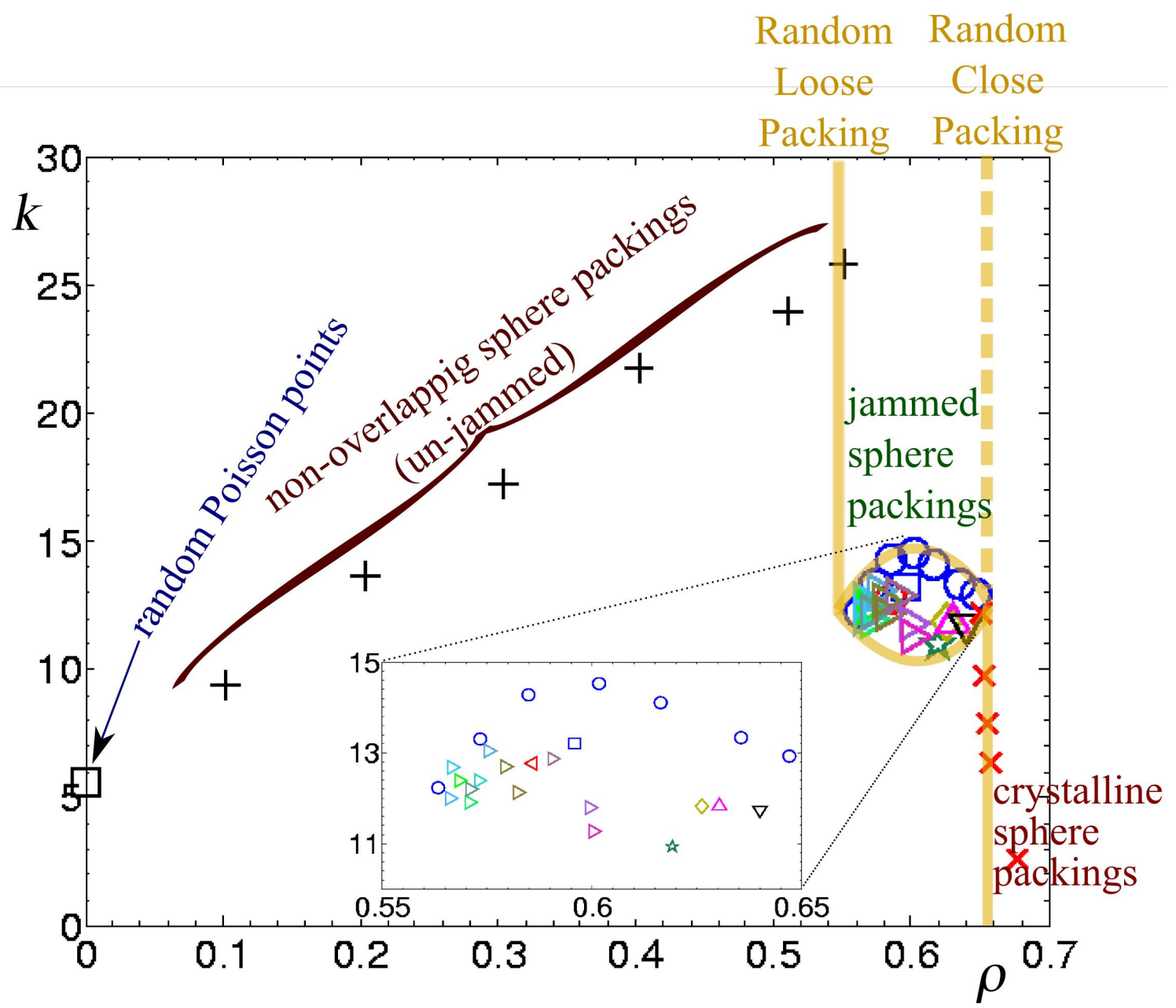

Figure 3. Behavior of $k$ calculated from the fluctuations of the Voronoï cells volumes by using $k=\left(\bar{V}-V_{\text {min }}\right)^{2} / \sigma_{v}^{2}($ Eq.7) vs. packing fraction $\rho$. Very sharp changes in the values of $k$ are observed at $\rho \sim 0.555$ and at $\rho \sim 0.645$. The ' + ' refer to simulations of un-jammed packings of spheres; the ' $\circ$ ' are jammed packings simulated by using different growth rates and ' $\times$ is a jammed sample with crystalline inclusions; the 6 symbols ' $\triangleleft, \square, \star, \diamond, \triangle, \nabla$ ' refer respectively to the 6 experimets A-F; whereas the symbols ' $\triangleright$ ' correspond to 12 experiments with glass spheres in water prepared by means of fluidized beds technique. ${ }^{4}$ 
which, in the formalism used in this paper, becomes

$$
S(Z)=k\left[1+\ln \left(\frac{\bar{V}-V_{\min }}{k \Lambda^{d}}\right)\right],
$$

leading to

$$
\beta_{g r}=\frac{\partial S(Z)}{\partial \bar{V}}=\chi^{-1}=\frac{k}{\bar{V}-V_{\min }} .
$$

The Edwards' compactivity $\beta_{g r}^{-17,8}$ is therefore the average free-volume per elementary cell $\chi=\left(\bar{V}-V_{\min }\right) / k$. This means that, in the present approach, the 'granular temperature' is a measure of the kind and the degree of space-partition into elementary cells. The volume fluctuations within the ergodic set can be directly calculated from Eq. 6 and one can verify that the correct relation between compactivity and volume fluctuations is attained:

$$
\chi^{2} \frac{\partial\langle V\rangle}{\partial \chi}=\left\langle(V-\langle V\rangle)^{2}\right\rangle
$$

From this equation, substituting Eq. 7, we obtain the following relation for the parameter $k$ :

$$
k=\frac{\partial\langle V\rangle}{\partial \chi}
$$

The parameter $k$ therefore mesures the amount of volume that must be added to the system in order to increase the compactivity by one 'granular degree'. The analogous quantity for molecular gasses is: $\partial E / \partial T$, which is the specific heat. In analogy with ordinary thermodynamics such 'specific heat' is expected to be sensitive to changes in the system's internal properties.

\section{EVIDENCE OF A PHASE TRANSITION FROM THE 'SPECIFIC HEAT' ANALYSIS}

We investigate the volume fluctuations at the level of a single grain. For this purpose we use the Voronoï partition where cells are associated with the portion of space closest to a grain center with respect to any other centre in the packing. $\mathrm{In}^{4}$ we have shown that the volume distribution of the Voronoï cells follows remarkably well the theoretical prediction $f(V, k)$ (Eq. 6) with $k$ in the range between 12.2 and 14.5. In $^{26}$ we show that the numerical simulations also reveal very good agreements with $f(V, k)$ but in this case the parameter $k$ spans over a larger interval $(9 \leq k \leq 25)$. The impressive fact of such agreements is that these systems are very different (ideal Newtonian spheres, acrylic beads in air and glass beads in water) and they are prepared in very different ways (pouring, tapping, fluid flows, shearing, hard-spheres molecular dynamics and soft-spheres Discrete Elment Method simulations). The fact that all these distributions follow the same law $f(V, k)$ suggests that there are universal properties that determine the packing configurations. On the other hand, the fact that different systems or different preparation methods yield to distributions with different values of $k$ indicates that this quantity might be an important parameter to control and characterize the system's properties.

Figure 3 reports the estimates for the parameter $k$ calculated for several computer simulations and experiments. The value at zero packing fraction $(k=5.586)$ was calculated analytically for random Poisson points in three dimensions. ${ }^{28,29}$ All the other values are instead calculated from the Voronoï cell volume fluctuations by using the relation $k=\left(\bar{V}-V_{\min }\right)^{2} / \sigma_{v}^{2}$ (Eq.7). Figure 3 shows that at low packing fractions, for non jammed configurations, the value of $k$ increases almost linearly with $\rho$. Then, it drastically decreases to values between 11 and 15 when the system gets into jammed configurations. The inset in the figure shows that there are differences in the values of $k$ for different systems and within the same system at different packing fractions. One can also note a rather sharp change in the experimental data occurring around $\rho \sim 0.6$ which might indicate some kind of transition at this packing fraction. Above the packing fraction $\sim 0.645(\mathrm{RCP})$, the packings contain partially crystallized regions and the change in the kind of structural organization is reveled by a sharp drop in the value of $k$ that eventually will go to zero at the crystalline limit. 


\section{CONCLUSIONS}

In this paper we provide two independent pieces of evidence showing transitions occurring in sphere packings at the Random Loose Packing and Random Close Packing limits. The first evidence is geometrical and it is acquired from the behavior of the fraction of common neighbours. In particular, we have observed that the fraction of couples with 3,5 and 2 or 4 common neighbours (Eq.1) have sharp changes occurring both at the RLP and RCP limits. Such changes indicate that rearrangements towards a different packing organization are occurring at these limiting packing fractions. The second evidence is acquired from a statistical mechanics study concerning the fluctuations in the local Voronoï volumes. We have found that all the samples investigated follow the same kind of statistical distribution $f(V, k)$ (Eq. 6) but they are characterized by different values of the quantity $k$. Sharp changes in the values of $k$ are observed when the packing fraction approaches the RLP and RCP limits. We have discussed that the quantity $k$ is analogous to the specific heat in ordinary thermodynamics. The sharp changes in this quantity indicate that an amount of available free volume is released or absorbed at these critical packing fractions, this in analogy with the 'latent heat' in thermochemistry.

\section{Acknowledgements}

Many thanks to T. Senden, M. Saadatfar, A. Sakellariou, A. Sheppard, A. Limaye for the tomographic data. This work was partially supported by the ARC discovery project DP0450292.

\section{REFERENCES}

1. T. Aste, D. Weaire, The Pursuit of Perfect Packing (Institute of Physics, Bristol, 2000)

2. J.D. Bernal, Proc. R.Soc. Lond. A280, 299 (1964)

3. M. Schröter, D.I. Goldman, H.L. Swinney, Phys. Rev. E. 71, 30301 (R) (2005)

4. T. Aste, T.D. Matteo, M. Saadatfar, T. Senden, M. Schröter, H.L. Swinney, Eur. Phys. Lett. 79, 240031 (2007)

5. J.D. Bernal, Nature 183, 141 (1959)

6. J.D. Bernal, J. Mason, Nature 188, 910 (1960)

7. S. Edwards, R. Oakeshott, Physica A 157, 1080 (1989)

8. A. Mehta, S.F. Edwards, Physica A 157, 1091 (1989)

9. A. Fierro, M. Nicodemi, A. Coniglio, Europhys. Lett. 59, 642 (2002)

10. H.A. Makse, J. Kurchan, Nature 415, 614 (2002)

11. R.P. Ojha, P.A. Lemieux, P.K. Dixon, A.J. Liu, D.J. Durian, Nature 427, 521 (2004)

12. P. Richard, M. Nicodemi, R. Delannay, P. Ribiere, D. Bideau, Nature Materials 4, 121 (2005)

13. M.P. Ciamarra, M. Nicodemi, A. Coniglio, preprint. (2006)

14. F. Lechenault, F. da Cruz, O. Dauchot, E. Bertin, J. Stat. Mech. P07009, 1742 (2006)

15. E.I. Corwin, H.M. Jaeger, S.R. Nagel1, Nature 435, 1075 (2005)

16. See, http://wwwrsphysse.anu.edu.au/granularmatter/

17. T. Aste, Phys. Rev. Lett. 96, 018002 (2006)

18. T. Aste, M. Saadatfar, T.J. Senden, Phys. Rev. E. 71, 061302 (2005)

19. T. Aste, M. Saadatfar, A. Sakellariou, T. Senden, Physica A 339, 16 (2004)

20. B.D. Lubachevsky, F.H. Stillinger, J. Stat. Phys. 60 (1990)

21. A. Donev, S. Torquato, F.H. Stillinger, J. Comput. Phys. 202, 737 (2005)

22. M. Skoge, A.D.F.H. Stillinger, S. Torquato, Phys. Rev. E. 74, 041127 (2006)

23. T. Aste, J. Phys.: Condens. Matter 17, S2361 (2005)

24. A.S. Clarke, H. Jónsson, Phys. Rev. E 47, 3975 (1993)

25. N.M. A.V. Anikeenko, Phys. Rev. Lett. 98, 235504 (2007)

26. T. Aste, T.D. Matteo, Phys. Rev. E, submitted (2007)

27. R.V. Hogg, A.T. Craig, Introduction to Mathematical Statistics (Macmillan, New York, 1978)

28. E.N. Gilbert, Ann. Math. Stat. 33, 958 (1962)

29. E. Pineda, P. Bruna, D. Crespo, Phys. Rev. E. 70, 0661191 (2004) 